\title{
Polarimetric, two-color, photon-counting laser altimeter measurements of forest canopy structure
}

\author{
David J. Harding ${ }^{* a}$, Philip W. Dabney and Susan Valett ${ }^{\mathrm{a}}$ \\ ${ }^{a}$ NASA Goddard Space Flight Center, 8800 Greenbelt Rd., Greenbelt, MD, USA 20771-2400 \\ *david.j.harding@nasa.gov; phone 1301 614-6503; fax 1301 614-6522; http:/science.gsfc.nasa.gov/698/personnel.html
}

\begin{abstract}
Laser altimeter measurements of forest stands with distinct structures and compositions have been acquired at $532 \mathrm{~nm}$ (green) and $1064 \mathrm{~nm}$ (near-infrared) wavelengths and parallel and perpendicular polarization states using the Slope Imaging Multi-polarization Photon Counting Lidar (SIMPL). The micropulse, single photon ranging measurement approach employed by SIMPL provides canopy structure measurements with high vertical and spatial resolution. Using a height distribution analysis method adapted from conventional, $1064 \mathrm{~nm}$, full-waveform lidar remote sensing, the sensitivity of two parameters commonly used for above-ground biomass estimation are compared as a function of wavelength. The results for the height of median energy (HOME) and canopy cover are for the most part very similar, indicating biomass estimations using lidars operating at green and near-infrared wavelengths will yield comparable estimates. The expected detection of increasing depolarization with depth into the canopies due to volume multiplescattering was not observed, possibly due to the small laser footprint and the small detector field of view used in the SIMPL instrument. The results of this work provide pathfinder information for NASA's ICESat-2 mission that will employ a $532 \mathrm{~nm}$, micropulse, photon counting laser altimeter.
\end{abstract}

Keywords: Canopy structure, lidar, polarimetry, micropulse, photon counting, waveform, SIMPL, ICESat

\section{INTRODUCTION}

Over the past 15 years lidar remote sensing has proven to be a highly effective method for characterization of forest canopy height and vertical structure ${ }^{[1,2]}$. Numerous papers have shown that this information is a strong predictor of above-ground biomass, providing an important means to quantify terrestrial ecosystem carbon storage and through time characterize sources and sinks of atmospheric $\mathrm{CO}_{2}$. A conventional laser altimeter measurement approach used for this purpose is medium-footprint, full-waveform recording typically done in the near-infrared (NIR) ${ }^{[3]}$. Micropulse, photon counting, typically done at green wavelengths, is a more recent approach with improved vertical resolution ${ }^{[3]}$. In this work we assess observations of forest canopy height and structure using this new approach, evaluating the results at near-infrared and green wavelengths and two polarization states. Specifically, we evaluate cumulative height distributions of detected photons adapting a method developed for conventional waveform measurements. We compare the height of median energy (HOME) and canopy cover, standard waveform metrics of canopy structure used in biomass estimation, to determine if they are wavelength sensitive and therefore a potential source of difference in biomass results obtained by NIR and green measurement approaches. We also assess if wavelength and polarization differences in the height distributions can provide information on the scattering of the pulsed laser energy within canopies that could yield information on the type and spatial organization of foliage, information potentially useful for differentiating tree species. Data used in this study was acquired using the Slope Imaging Multi-polarization Photon-counting Lidar (SIMPL) flown over three forested sites having a diversity of canopy vertical structures.

\subsection{Medium-footprint, full-waveform lidar}

Full-waveform systems include three laser altimeters developed by NASA, the airborne Scanning Lidar Imager of Canopies by Echo Recovery (SLICER) ${ }^{[4]}$, the airborne Laser Vegetation Imaging Sensor (LVIS) ${ }^{[5]}$ and the Geoscience Laser Altimeter System (GLAS) ${ }^{[6,7]}$ flown aboard the Ice Cloud and Land Elevation Satellite (ICESat) ${ }^{[8.9]}$. Those systems utilize a single pulse analog measurement approach depicted schematically in Figure 1. The systems operate at $1064 \mathrm{~nm}$ (NIR) with pulse widths in the range 7 to $10 \mathrm{nsec}$ full-width half-max (FWHM), equivalent to 1 to $1.5 \mathrm{~m}$. The pulses illuminate footprints on the Earth's surface with diameters varying between $10 \mathrm{~m}$ for SLICER up to $70 \mathrm{~m}$ for GLAS. 
Reflected laser energy is detected using a Silicon Avalanche Photodiode (Si:APD) that converts received energy to an output voltage time series that is digitized, nominally with $1 \mathrm{nsec}(15 \mathrm{~cm})$ sampling, providing a lidar waveform. The waveform is a measure of the within-footprint vertical distribution of canopy surfaces (foliage, stems, branches) and the underlying ground where illuminated by the laser pulse ${ }^{[4]}$. High pulse energies are used in order to detect sufficient signal $(1,000 \mathrm{~s}$ of photons) above the Si:APD noise floor operating from medium altitude airborne platforms $(5 \mathrm{~mJ}$ from $10 \mathrm{~km}$ for LVIS) to spaceflight altitudes $(50 \mathrm{~mJ}$ from $600 \mathrm{~km}$ for ICESat). This signal level is necessary to fully characterize the canopy structure and to detect the ground surface beneath dense, closed canopies, both of which are necessary to determine canopy height. Because of the high pulse energies, the pulse rate must be low for a practical laser transmitter implementation ( $500 \mathrm{~Hz}$ for LVIS, $40 \mathrm{~Hz}$ for ICESat) thereby limiting their spatial sampling and resolution. LVIS acquires contiguous footprints, nominally $20 \mathrm{~m}$ in diameter, across a several $\mathrm{km}$ wide swath. ICESat samples along a single profile, nominally with $50 \mathrm{~m}$ diameter footprints spaced apart by $170 \mathrm{~m}$. In addition to limited spatial resolution, the relatively broad pulse width acts as a low pass filter, thereby smoothing the waveform and limiting the vertical resolution of canopy features. This also broadens the return from the ground and reduces its amplitude thus making its detection more difficult ${ }^{[3]}$.

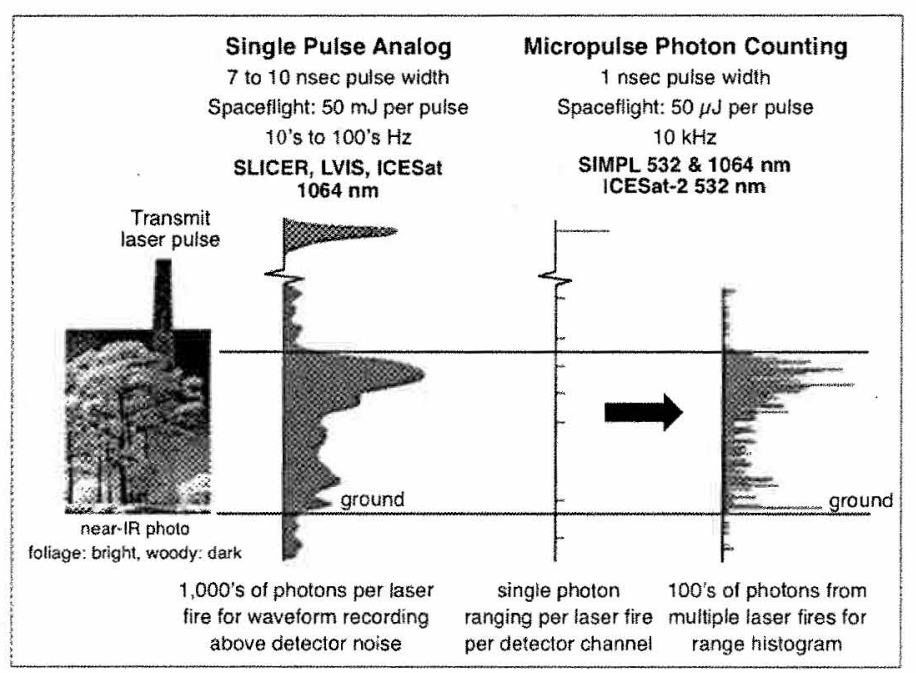

Fig. 1. Single pulse analog and micropulse photon counting measurement approaches for characterization of forest canopy height and vertical structure, adapted from ${ }^{[3]}$.

One method to characterize canopy structure and estimate above-ground biomass using conventional lidar waveforms

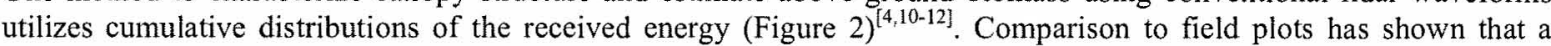
multi-parameter, linear regression using several waveform-derived parameters is a strong predictor of field-measured above-ground biomass. An early approach ${ }^{[10-11]}$ used four parameters: the canopy height (distance from the center of the ground return to the highest detected signal above a threshold), the height of median energy (HOME, the distance from the ground return to the $50^{\text {th }} \%$ ), the ratio of the two heights, and the received energy of the ground return divided by that of the canopy (a measure of canopy cover, the percentage of ground covered by a vertical projection of the canopy surfaces). Other studies use the ground received energy divided by the total energy as the measure of cover ${ }^{[4]}$. This is the criteria used in this study. HOME is sensitive to changes in both the vertical arrangement of canopy elements and the canopy cover ${ }^{[10]}$. As observed by a lidar, the cover is a function of both plant area and the retro-reflectance of the ground relative to that of the canopy at the laser wavelength ${ }^{[4]}$, with higher relative ground retro-reflectance resulting in a lower cover result. The enhanced-amplitude, "hot spot" retro-reflectance at $0^{\circ}$ phase angle is the applicable reflectance for lidar instruments because they have parallel illumination and view angles ${ }^{[4]}$.

\subsection{Micropulse, photon counting lidar}

To address the analog waveform limitations of low detector sensitivity and restricted vertical and spatial resolution an alternative measurement approach has been developed. Micropulse, photon counting (Figure 1) uses a high repetition 


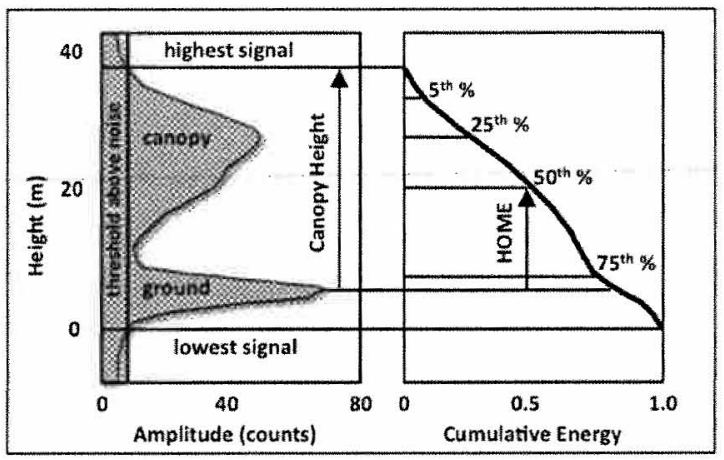

Fig. 2. A lidar waveform and the derived cumulative distribution indicating percentile heights of received energy, including the $50^{\text {th }} \%$ height of median energy (HOME) (adapted from https://lvis.gsfe.nasa.gov).

rate laser transmitter, nominally at $10 \mathrm{kHz}$ with a $\sim 1 \mathrm{nsec}(15 \mathrm{~cm})$ pulse width and low pulse energy, $\sim 1 / 1,000$ that of a traditional system with comparable performance ${ }^{[3,13-16]}$. Typically a photomultiple tube (PMT) in Geiger mode with high sensitivity at visible wavelengths is used to detect the arrival of back-scattered single photons at $532 \mathrm{~nm}$ that are transmitted by frequency doubling a $1064 \mathrm{~nm}$ laser. To increase the count rate of single photons per laser fire a PMT array with multiple detector channels is often used. The arrival time of the single photons is nominally resolved to 0.1 nsec $(1.5 \mathrm{~cm})$ using an event timer. The round-trip travel time between the laser fire and photon detection event provides the ranging distance to the reflecting target. By accumulating the detected photons for a sequence of laser fires into a range histogram a measurement analogous to a waveform is generated ${ }^{[3]}$. Because the micropulse width is $\sim 10 \mathrm{x}$ narrower and the timing precision is $\sim 10 \mathrm{x}$ better, a measurement with substantially better vertical resolution than a conventional waveform is achieved. A key benefit is that returns from canopy layers and the ground are narrower and therefore higher in amplitude making their detection more reliable. Fewer detected photons are therefore required to achieve a measurement of comparable sensitivity. In photon counting systems the noise floor of the detectors is essentially zero and the dominant noise source becomes solar background illumination, which can hinder the detection of weak returns. To minimize the noise rate a small detector instantaneous field of view (IFOV) only a few times larger than the footprint is used, and narrow band spectral filtering further reduces the noise. NASA's ICESat-2 mission, a follow-on to ICESat planned for launch in 2016, will use this measurement approach ${ }^{[17]}$. Whereas ICESat employed a single laser beam, ICESat-2 will measure six profiles in a "push broom" configuration using a diffractive optical element (DOE) to split the output of a single laser into six beams. The beams are configured for observing ice sheet elevation change by precisely repeating measurement tracks through time. The planned design will have three tracks separated by $3 \mathrm{~km}$ for spatial coverage, with each track comprised of two profiles spaced cross-track by $<100 \mathrm{~m}$ in order to separate elevation differences due to slope from actual elevation change. Each profile will be sampled with $10 \mathrm{~m}$ diameter laser footprints and $0.7 \mathrm{~m}$ spacing between footprints, providing substantially better spatial resolution than that of ICESat.

\section{SLOPE IMAGING MULTI-POLARIZATION PHOTON-COUNTING LIDAR}

SIMPL is an airborne, micropulse, photon counting laser altimeter developed through the NASA Earth Science Technology Office Instrument Incubator Program. It incorporates a variety of advanced technologies in order to demonstrate measurement approaches of potential benefit for improved, more efficient spaceflight laser altimeter missions including ICESat-2. Instrument details are documented elsewhere ${ }^{[18]}$. In summary, SIMPL incorporates beam splitting of a micropulse laser, single-photon ranging and polarimetry technologies at green (532 nm) and NIR (1064 nm) wavelengths. The basic measurement approach and the micropulse transmitter characteristics are similar to that described above. Like ICESat-2, the measurement configuration is a "push-broom" but with four beams spaced apart equally perpendicular to the flight direction. However, because use of a DOE would separate the transmit paths of the two colors, a dichroic beam splitter based on bi-refringent calcite crystals was developed to preserve co-aligned colors. The splitter also maintains transmit pulses that are plane polarized. For each beam the received energy is separated into 
the two colors and each color is further separated into energy with polarization states parallel $(\|)$ and perpendicular $(\Lambda)$ to the transmitted polarization, yielding four channels per beam and a total of 16 channels. Traditional PMT detectors are not used because they are insensitive at NIR wavelengths. Instead, for each channel SIMPL employs a Single Photon Counting Module (SPCM) ${ }^{[19]}$ that has sensitivity at both visible and NIR wavelengths. The probability of detecting (PD) a photon per laser fire is kept below $30 \%$ for each channel, by adjusting mechanical irises and flight altitude, in order to minimize biasing the ranging distance and depolarization measurements that can be introduced at high detection rates due to the long, $\sim 50 \mathrm{nsec}(7.5 \mathrm{~m})$ dead-time of the SPCM detectors ${ }^{[18,19]}$.

SIMPL's measurements provide information about surface elevation, roughness and slope as well as scattering properties used to differentiate surface properties and types. By measuring the depolarization of the plane-polarized transmit pulses at the two wavelengths SIMPL has a unique capability to differentiate surface types, building upon prior non-ranging measurements of laser depolarization at 355,532 and $1064 \mathrm{~nm}^{[20,21]}$. By adding the ranging component, SIMPL provides a means to investigate the vertical distribution of optical scattering properties to better understand the interaction of pulsed laser energy with surface targets. The depolarization ratio ( $L / 11$ ) is sensitive to the proportions of specular reflection and surface and volume scattering, and is a function of wavelength. Upon single scattering from a surface the reflected energy remains parallel to that of the plane-polarized laser pulse. As multiple scattering increases the fraction of the energy converted to the perpendicular state increases as does the depolarization ratio. Prior work using data acquired over Lake Erie ice cover demonstrated differentiation of open water, snow and several ice facies as well as differences in the depth of laser pulse penetration ${ }^{[18]}$, results of relevance for planned ICESat- 2 measurements of sea ice thickness ${ }^{[17]}$. For canopies, the degree of depolarization is a function of wavelength and the reflectance phase function, transmission, size and spacing of foliage and woody components and the specular character of waxy broadleaf coatings, if present ${ }^{[20]}$. Ground depolarization is a function of wavelength, the type of ground-cover and its retro-reflectance and roughness.

\subsection{Forest canopy data collection}

SIMPL data was collected over four forest sites using the NASA Lear-25 operated by Glenn Research Center in late July and early August, 2010: the Smithsonian Environmental Research Center (SERC), MD where the forest structure has been documented by extensive ground observations ${ }^{[22]}$; two locations within the New Jersey Pine Barrens (PB) (Cedar Bridge and Silas Little) centered on USDA Forest Inventory and Analysis plots; and a site in the Huron National Forest (HNF), MI. Short segments of the data acquired on one flight line at each site are shown in Figures 3-5. The SERC and Pine Barrens sites are being used by the ICESat-2 project for measurement validation and algorithm development. At each of these sites $15 \mathrm{~km}$ data segments were collected on 6 flight lines that repeated lines used earlier in 2010 by a swath mapping, $532 \mathrm{~nm}$, micropulse, photon counting lidar operated by Sigma Space Corp. Two lines were flown and then repeated at the HNF site that was selected because it consists of single-species, managed pine stands that provided distinct changes in forest height and structure. At the nominal flight altitude of $2.3 \mathrm{~km}$ above ground level (AGL) at SERC, the spacing between the beams was $5 \mathrm{~m}$, the footprint diameter was $0.2 \mathrm{~m}$ and the detector IFOV was $0.6 \mathrm{~m}$. These dimensions are small because SIMPL's optical design was predicated on eventual flight on the International Space Station at a nominal altitude of $360 \mathrm{~km}$, from which the beam spacing and footprint size would be $800 \mathrm{~m}$ and $30 \mathrm{~m}$, respectively. The nominal altitudes at the $\mathrm{PB}$ and $\mathrm{HNF}$ sites were $2.0 \mathrm{~km}$ and $1.8 \mathrm{~km} \mathrm{AGL}$, respectively. At the laser fire rate of $11.4 \mathrm{kHz}$ and a nominal aircraft ground speed of $100 \mathrm{~m} / \mathrm{sec}$ and PD between $5 \%$ and $15 \%$, the typical density of signal photons detected was between 5 and 15 per $\mathrm{m}$. SIMPL's micropulse transmitter normally has a $2.7 \mathrm{nsec}$ FWHM after pulse occurring $8 \mathrm{nsec}(1.2 \mathrm{~m})$ after the primary pulse. The amplitude of the after pulse at $1064 \mathrm{~nm}$ is about $20 \%$ that of the main pulse. The amplitude is reduced to about $5 \%$ of the main pulse at 532 nm due to the process of frequency doubling. The after pulse was present during the PB and HNF flights. The after pulse amplitude was greatly reduced during the SERC flight, inferred to be due to alteration of the laser operating state by high pre-flight ambient temperatures in the aircraft.

\subsection{Results}

Plotting ranging distance as a function of acquisition time for each detected photon yields single-photon "point clouds" for each of SIMPL's 16 channels, providing a detailed depiction of the two dimensional structure of the forest stands and a profile of the underlying ground topography. Figures 3-5 show examples for the third beam's $532 \mathrm{~nm} \|$ channel. The point density for the SERC example appears lower than the other two sites because a shorter flight segment is depicted, thereby expanding its horizontal axis. Undulations in the range to the ground are a combination of topographic relief and changes in aircraft altitude and attitude. Three stands at each site were selected to derive and evaluate range histograms. Stands with distinct canopy height, structure and cover were selected based on visual examination of the point cloud 
profiles and associated imagery. At SERC the stand types are: A) $\sim 115$ year old mixed broad-leaf hardwoods with high canopy cover, B) $\sim 70$ broad-leaf tulip poplar and sweet gum with moderate canopy cover, and C) 15 year loblolly pine, selectively logged in 2007 , with low canopy cover ${ }^{[23]}$. At PB specific information on the selected stands is not available. The region has naturally occurring mixed pine and deciduous cover. At HRN the stand types are: A) a recent jack pine plantation with high canopy cover, B) a red pine plantation established in 1942 with moderate canopy cover, and C) the 1942 red pine plantation which has had low thinning applied to reduce under-story tree density ${ }^{[24]}$. Distinct changes in forest height and vertical structure are especially apparent in the HRN point cloud at boundaries between the stands of different age and silviculture management practices (Figure 5). To the right of stand A is a very young jack pine plantation stand into which is cut trapezoidal gaps as a part of a program of habitat maintenance for the endangered Kirtland's Warbler. The gaps in the short stature pines are observed in the point cloud. To the left of stand B is a recent
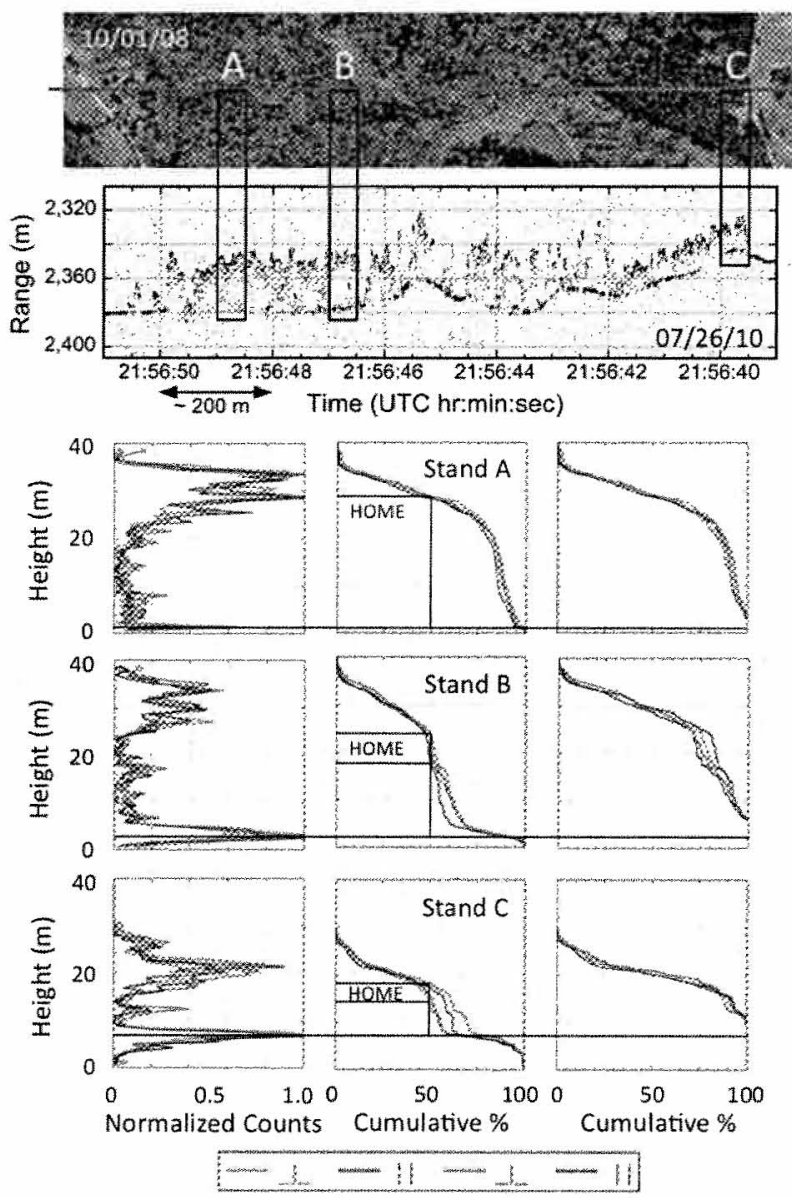

Fig. 3. Results for SERC, MD data acquired on 07/26/10. Top: SIMPL profile track (red) displayed on an aerial photograph from Google Earth acquired on 10/01/08. The location along the track for the three selected stands is indicated by the black segments, each of which are $\sim 50 \mathrm{~m}$ in length. At this scale the small cross-track separation between the four beams is not apparent. Middle: single photon point cloud for the $532 \mathrm{~nm}$ parallel channel on beam 3 with the stand location indicated by the boxes. Bottom: beam 3 height distributions for the three stands showing number of received photons, binned at $0.5 \mathrm{~m}$, with each channel normalized by its peak amplitude (left); full cumulative distributions (FCDs) of total received photons, excluding the laser after-pulse, with HOME indicated showing its height range where the channel distributions diverge (center); and canopy cumulative distributions (CCDs) of received photons excluding the ground return (right). Colors represent the $1064 \mathrm{~nm}$ perpendicular (orange) and parallel (red) channels, and the $532 \mathrm{~nm}$ perpendicular (light green) and parallel (dark green) channels. 


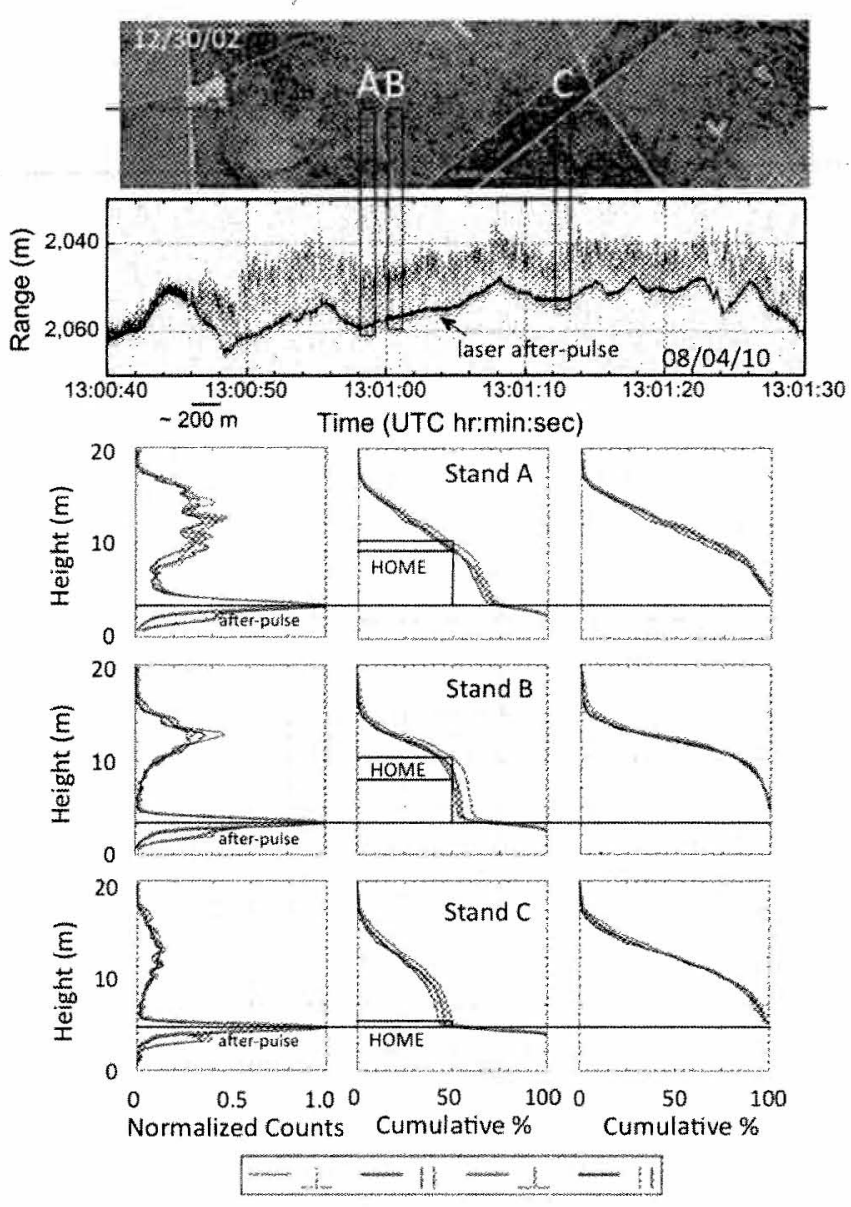

Fig. 4. Results for the Cedar Bridge Pine Barrens, NJ data acquired on 08/04/10. Top: SIMPL profile track (red) displayed on an aerial photograph from Google Earth acquired on 12/30/02. The stand segments are $100 \mathrm{~m}$ long. See Figure 3 caption for explanation.

clear cut. Seed trees left standing to re-populate the site are observed in the point cloud, attesting to the high horizontal resolution achieved by this small laser footprint, single photon data.

In order to evaluate canopy vertical structure range histograms were computed by aggregating all detected photons in each of the 9 selected stands for each of the four channels on beam 3. The range histograms are presented in three ways in Figures 3-5, height distributions normalized by peak amplitude, full cumulative distributions (FCD) including the canopy and ground returns (excluding the laser after-pulse), and canopy cumulative distributions (CCD) (excluding the ground return). HOME is indicated on the FCDs, determined in the same manner as for conventional lidar waveforms. Where the distributions for the four channels at a site diverge, the height range of HOME is shown. The primary features of the individual stand height distributions for all four channels are very similar including: (1) the stand height, (2) the slope of the leading signal up to the initial canopy peak, where a greater slope corresponds to greater openness of the outer canopy surface ${ }^{[4]},(3)$ the crown depth indicated by the height range of the broad, primary canopy signal, (4) the location of prominent layers in the crown and the under-story indicated by narrow, secondary peaks, (5) the summed amplitude of the ground return relative to that of the total return which is the reflectance-weighted measure of canopy cover $^{[4]},(6)$ segments in the under-story lacking significant cover (canopy vertical gaps) where the signal approaches zero, and (7) the width of the ground return which is a measure of the relief within the stand due to slope and/or roughness ${ }^{[7]}$. The consistency of the narrow peaks between all four channels for a stand indicates that these are real 
attributes of the stand structure, not noise, and demonstrates the high vertical resolution of the micropulse, photon counting technique even at this relatively coarse binning of $0.5 \mathrm{~m}$. Differences between these height distribution attributes document significant structural differences between the stands.

The similarity of a stand's height distributions for the four channels leads to significant similarity in that stand's FCDs, especially so for SERC stand A and HNF stand A and B; yielding a common HOME for all channels. The other stands show some divergence in the FCDs to varying degrees, with channels displaced to the right having a greater amount of received photons occurring above a given height. For SERC stand C and PB stands B and C the $1064 \mathrm{~nm} \|$ channel is left-most and the $532 \mathrm{~nm} \_$__ channel is displaced most to the right. For HNF stand C $532 \mathrm{~nm} \|$ is left-most and $1064 \mathrm{~nm}$ $\ldots$ _ is displaced most to the right. For the other stands the separations are less well defined. The divergence between channels leads to within-stand differences in HOME up to $6 \mathrm{~m}$ (SERC stand B) in an absolute sense and up to $20 \%$ as a fraction of stand height (SERC stand $\mathrm{C}$ and $\mathrm{PB}$ stand $\mathrm{B}$ ). Although HNF stand $\mathrm{C}$ has a significant divergence of the channels, HOME is constant because the separation occurs at cumulative percentages greater than $50 \%$. At some percentiles the corresponding heights can differ substantially. The largest case is HNF stand C where the $532 \mathrm{~nm} \| 70^{\text {th }}$ $\%$ height is essentially zero and is $9 \mathrm{~m}$ for $1064 \mathrm{~nm} \_$_, which is $\sim 50 \%$ of the stand height.
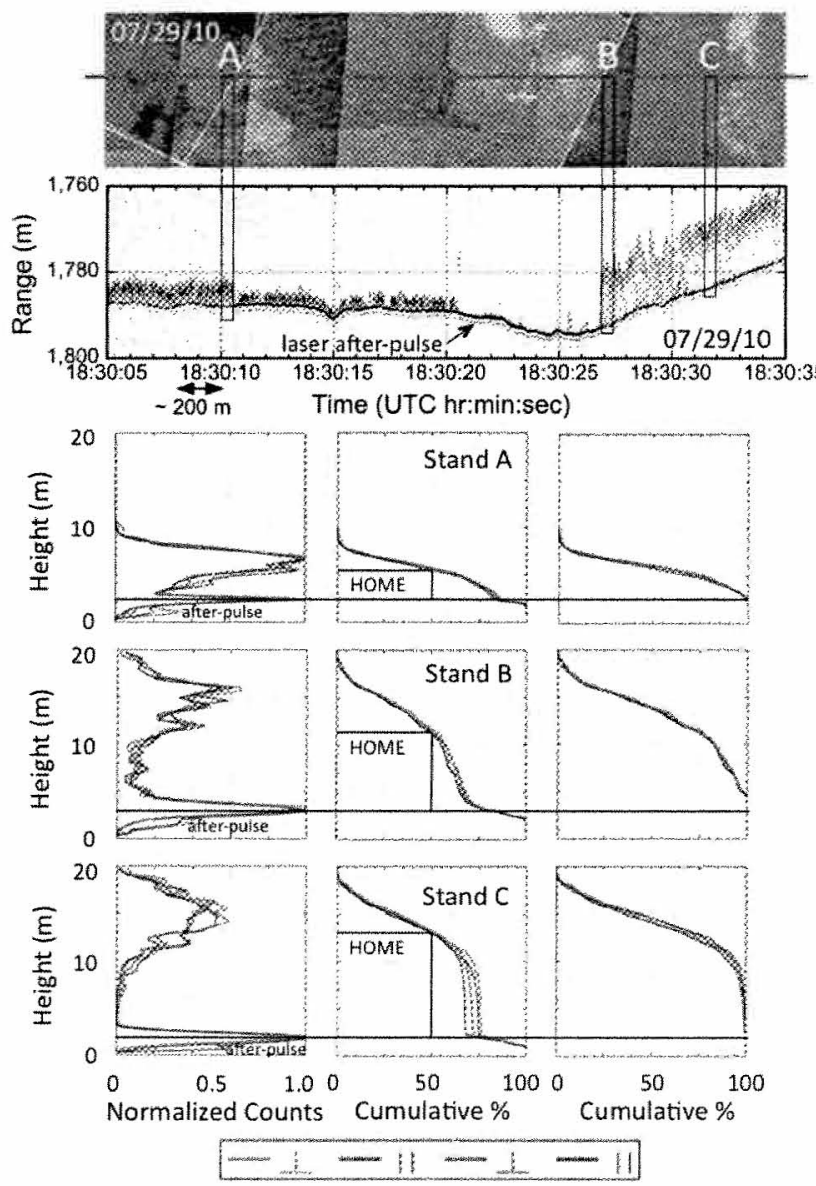

Fig. 5. Results for the Huron National Forest, MI data acquired on 07/29/10. Top: SIMPL profile track (red) displayed on a mosaic of nadir-viewing video frames acquired in-flight. The stand segments are $\sim 100 \mathrm{~m}$ long. See Figure 3 caption for explanation. 
On the FCDs the reflectance-weighted canopy cover is indicated by the percentile where the distributions reach the start of the ground return height. Divergence between the channels for a stand results in between-wavelength differences in the cover estimate that are for the most part small, below 10\%. SERC stand C exhibits the largest difference, with 55\% cover for the $1064 \mathrm{~nm}$ channels and $70 \%$ cover for the $532 \mathrm{~nm} \_$channel. Differences in the FCD intercept with the ground return document significant between-stand differences in their cover. For example, the three stands at PB do not differ markedly in appearance in the point cloud plot because their vertical structure is fairly similar, as indicated by the shape of their height distributions, but the cover ranges from $70 \%$ (stand A) to $45 \%$ (stand C).

The generally close agreement between the $1064 \mathrm{~nm}$ and $532 \mathrm{~nm}$ HOME and canopy cover metrics documents that these canopy parameters, at least for these stands, are rather insensitive to wavelength differences, and indicates that biomass estimation using NIR and green laser altimeters will be comparable. This suggests that the $532 \mathrm{~nm}$ ICESat-2 observations can be interpreted using similar biomass estimation relationships that have been developed for the $1064 \mathrm{~nm}$ SLICER, LVIS and GLAS instruments.

In all the cases but one the within-stand channels CCDs are essentially the same. For SERC stand B there is some separation of the channels at high cumulative percentiles. Because the ground return signal is removed from the CCDs, they are a more direct means than the FCDs to compare canopy vertical structure, independent of cover. For example, the PB CCDs for stands A and B are very similar despite their differences in cover, consistent with their similar crown shapes in the height distributions.

\subsection{Discussion}

This work is the first of its kind, examining dual-wavelength, polarimetric lidar observations with high spatial and vertical resolution acquired through a diversity of canopies. As such, the results could not be fully anticipated. However, we had several expectations. We assumed that increasing multiple scattering with depth into canopies would cause an increasing amount of _ _ received energy as compared to $\|$, similar to water column volume scattering observed in SIMPL data ${ }^{[23]}$. Therefore, we expected to see divergence between these channels in the CCDs progressing into the canopies with the $\|$ signal displaced to the right indicating that is preferentially occurring higher in the canopy than the

$\perp$ signal. Furthermore, increasing reflectance generally increases the degree of multiple scattering so we expected that the effect would be more pronounced at $1064 \mathrm{~nm}$ due to the substantially higher foliage reflectance in the NIR compared to the green, often by as much as a factor of $10 \mathrm{x}$. Therefore, the similarity across channels in the CCDs is unexpected. In the one case where divergence is seen (SERC stand B) the $532 \mathrm{~nm}$ result conforms to our expectation but the opposite relationship holds true for $1064 \mathrm{~nm}$. Several factors could be diminishing detection of a depolarization signal with depth into the canopies. The laser footprint is very small, $\leq 0.2 \mathrm{~m}$. If it is small with respect to gaps in the canopy the first encounter with a plant surface, where single scattering dominates, could occur deep within the canopy thereby reducing the occurrence of multiple scattering at lower depths. In addition, the very small detector IFOV used to limit the solar background noise rate could be excluding a substantial portion of the multiply scattered photons. With decreasing IFOV the return signal becomes increasingly dominated by the single-scattered direct backscatter from the first encountered surfaces. The degree to which multiply scattered photons are excluded depends on the path length between scattering events, controlled by foliage shape and spacing. To better understand these unexpected results we are initiating modeling work to evaluate the effect of instrument parameters on the detection of canopy depolarization.

Because the CCDs are insensitive to channel differences, we interpret the divergence of channels within the FCDs to be due to changes in the relative magnitudes of the canopy and ground retro-reflectance between channels. For the most common case of the $532 \mathrm{~nm}$ _ channel being most displace to the right, that indicates that the ground is least reflective relative to the canopy for this wavelength and polarization state. The degree of channel separation for a stand is a measure of the magnitude of ground versus canopy reflectance differences. These differences in the reflectance ratios provides information on the optical scattering properties of the canopy and ground cover. The changes in the order of channel displacements between stands indicates there are changes in canopy and/or ground cover type. We will investigate the potential use of this information for differentiating canopy and ground cover types as a part of our modeling work.

The number of stands we have assessed in this work is limited to nine. Further work examining more examples of these stand types at the sites is needed to ascertain if the relationships observed are consistent. In addition, the stands examined are mostly rather open, pine-dominated forest cover with only two closed-canopy, broadleaf forests included in the assessment. More broadleaf forest cover types with a greater diversity of stand structures will be included in future work. Lastly, the wavelength comparison of HOME and cover was done using micropulse, photon counting for 
each. We do not expect that results will differ substantially between conventional waveforms and photon range histograms because both are observing the travel time of photons in an unbiased manner. However, this assumption needs to be confirmed by direct comparison of NIR waveforms and green photon counting height distributions.

\section{CONCLUSION}

This work provides the first detailed examination of forest canopy structure as a function of two laser altimeter wavelengths and two polarization states. Analysis of results for nine stands with different age, structure and tree composition at three sites in Maryland, New Jersey and Michigan shows that the SIMPL instrument's implementation of the micropulse, photon counting approach provides structure measurements with high vertical and spatial resolution. The well-resolved forest cover structure portrayed in single photon point clouds enables selection of distinct stands for subsequent analysis using height distributions of received photons analogous to conventional lidar waveforms. The primary attributes of the examined stands are similar at $1064 \mathrm{~nm}$ and $532 \mathrm{~nm}$, in particular for the HOME and cover metrics commonly used for above-ground biomass estimation, indicating that NIR and green lidar systems will provide comparable estimations. Significant differences expected in height distributions as a function of wavelength and polarization state, due to within-canopy volume scattering causing increasing depolarization with depth, were observed for only one stand. We speculate that the small lidar footprint and/or small detector IFOV are minimizing detection of multiply-scattered photons with polarization perpendicular to that of the transmitted laser pulses. Modeling work is being initiated to assess instrument parameter influence on the detection of multiple-scattering. We infer differences in canopy cover estimates as a function of wavelength and polarization are related to differences in the ground reflectance relative to that of the canopy between the four channels. Our modeling work will assess the potential for differentiation of canopy and ground types based on the differences in these reflectance ratios. This work is providing pathfinder results to better understand and interpret the non-polarimetric, $532 \mathrm{~nm}$ micropulse, photon counting data that will be acquired by the NASA ICESat-2 mission, scheduled for launch in 2016.

\section{Acknowledgements}

We thank Edward Hicks, Chris Shuman, Aleksey Vasilyev and Anthony Yu from NASA Goddard Space Flight Center for their assistance installing and operating the SIMPL instrumentation, the NASA Ames Airborne Sciences Program for providing the Applanix POS/AV system and Bob Billings for its installation, the personnel of Glenn Research Center's Airborne Flight Operations for provision of the NASA Lear-25, especially lead pilot Kurt Blankenship and chief mechanic Steve Hughell, and the Goddard Space Flight Center Director's Discretionary Fund and the ICESat-2 Project for their financial support.

\section{REFERENCES}

[1] Lefsky, M.A., Cohen, W.B., Parker, G.G. and Harding, D.J., "Lidar remote sensing for ecosystem studies," Bioscience 52(1), 19-30 (2002).

[2] Bergen, K.M., Goetz, S.J., Dubayah, R.O., Henebry, G.M., Hunsaker, C.T., Imhoff, M.L., Nelson, R.F., Parker, G.G. and Radeloff, V.C., "Remote sensing of vegetation 3-D structure for biodiversity and habitat: review and implications for lidar and radar spaceborne missions," J. Geophys. Res. Biogeosci. 114, G00E06, DOI: 10.1029/2008JG000883 (2009).

[3] Harding, D.J., "Pulsed laser altimeter ranging techniques and implications for terrain mapping," in Topographic Laser Ranging and Scanning: Principles and Processing, Jie Shan and Charles Toth, eds., CRC Press, Taylor \& Francis Group, pp. 173 - 194 (2010).

[4] Harding, D. J., M.A. Lefsky, G.G. Parker, J.B. Blair, "Laser altimeter canopy height profiles: methods and validation for deciduous, broadleaf forests," Rem. Sens. Environ. 76(3), 283-297 (2001).

[5] Blair, J.B., D. L. Rabine and M. A. Hofton, "The Laser Vegetation Imaging Sensor (LVIS): a medium-altitude, digitization-only, airborne laser altimeter for mapping vegetation and topography," ISPRS J. Photogram. Rem. Sens. $54,115-122(1999)$. 
[6] Abshire J.B., X. Sun, H. Riris, J.M. Sirota, J.F. McGarry, S. Palm, D. Yi, P. Liiva, "Geoscience Laser Altimeter System (GLAS) on the ICESat mission: on-orbit measurement performance," Geophys. Res. Lett. 32, L21S02 doi:10.1029/2005GL024028 (2005).

[7] Harding, D.J., and C.C. Carabajal, "ICESat waveform measurements of within-footprint topographic relief and vegetation vertical structure," Gephys. Res. Lett. 32, L21S10 10.1029/2005GL023471 (2005).

[8] Zwally, H.J., R. Schutz, W. Abdalati, J. Abshire, C. Bentley, J. Bufton, D. Harding, T. Herring, B. Minster, J. Spinhirne and R. Thomas, "ICESat's laser measurements of polar ice, atmosphere, ocean, and land," Journal of Geodynamics 34(3-4), 405-445 (2002).

[9] Schutz, B.E., Zwally, H.J., Shuman, C.A., Hancock, D. and DiMarzio, J.P., "Overview of the ICESat mission," Geophys. Res. Lett. 32, L21S01, DOI: 10.1029/2005GL024009 (2005).

[10] Drake, J.B., Dubayah, R.O., Clark, D.B., Knox, R.G., Blair, J.B., Hofton, M.A., Chazdon, R.L., Weishampel, J.F., Prince, S.D., "Estimation of tropical forest structural characteristics using large-footprint lidar," Rem. Sens. Environ. 79(2-3), 305-319 (2002).

[11] Drake, J.B., Dubayah, R.O., Knox, R,G., Clark, D.B., Blair, J.B., "Sensitivity of large-footprint lidar to canopy structure and biomass in a neotropical rainforest," Rem. Sens. Environ. 81(2-3), 378-392 (2002).

[12] Dubayah, R.O., Sheldon, S.L., Clark, D.B., Hofton, M.A., Blair, J.B., Hurtt, G.C. and Chazdon, R.L., "Estimation of tropical forest height and biomass dynamics using lidar remote sensing at La Selva, Costa Rica," J. Geophy. Res. Biogeosci. 115, G00E09, DOI: 10.1029/2009JG000933 (2010).

[13] Degnan, J.J., J. McGarry, T. Zagwodzki, P. Dabney, J. Geiger, R. Chabot, C. Steggerda, J. Marzouk and A. Chu, "Design and performance of an airborne multikilohertz photon-counting, microlaser altimeter," Proc. Land Surface Mapping and Characterization using Laser Altimetry, Int. Arch. Photogramm. Rem. Sens. XXXIV3-W4, 9-16 (2001).

[14] Degnan, J.J., "Photon-counting multikilohertz microlaser altimeters for airborne and spaceborne topographic measurements," J. Geodyn. 34(3-4), 503-549 (2002).

[15] Carter, W.E., R.L. Shrestha and K.C. Slatton, "Photon counting airborne laser swath mapping (PC-ALSM)," in Gravity, Geoid and Space Missions, C. Jekeli, L. Bastos and J. Fernandes (Eds.), IAG International Association of Geodesy Symposia 129, 214 - 217 (2004).

[16] Cossio, T., C. Slatton, W. Carter, K. Shrestha and D. Harding, "Predicting topographic and bathymetric measurement performance for low-SNR airborne lidar," IEEE Trans. Geosci. Rem. Sens., 47(7), 2298 - 2315 (2009).

[17] Abdalati, W., H.J. Zwally, R, Bindschadler, B, Csatho, S.L. Farrell, H.A. Fricker, D. Harding, R. Kwok, M. Lefsky, T. Markus, A. Marshak, T. Neumann, S. Palm, B. Schutz, B. Smith, J. Spinhirne and C. Webb, "The ICESat-2 laser altimetry mission," Proc. IEEE 98(5), 735-75I (2010).

[18] Dabney, P., Harding, D., Abshire, J., Huss, T., Jodor, G., Machan, R., Marzouk, J., Rush, K., Seas, A., Shuman, C., Sun, X., Valett, S., Vasilyev, A., Yu, A. and Zheng, Y., "The Slope Imaging Multi-polarization Photon-counting Lidar: development and performance results," Proc. Int. Geosci. Rem. Sens. Symp., 11686732, DOI 10.1109/IGARSS.2010.5650862, 253-256 (2010).

[19] Sun, X., Krainak, M.A., Abshire, J.B., Spinhirne, J.D., Trottier, C., Davies, M., Dautet, H., Allan, G.R., Lukemire, A.T. and Vandiver, J.C., "Space-qualified silicon avalanche-photodiode single-photon-counting modules," J. Modern Optics, 51(9), 1333-1350 (2004).

[20] Kalshoven, Jr., J.E. and P.W. Dabney, "Remote sensing of the Earth's surface using an airborne polarized laser," IEEE Trans. Geosci. Remote Sensing 31, 438-446 (1993).

[21] Kalshoven, Jr., J.E., M.R. Tierney, Jr., C.S. T. Daughtry and J.E. McMurtrey III, "Remote sensing of crop parameters with a polarized, frequency-doubled Nd:YAG laser," Appl. Opt. 34, 2745-2749 (1995).

[22] Parker, G.G., and Tibbs, D.J., "Structural phenology of the leaf community in the canopy of a Liriodendron tulipifera L. forest in Maryland, USA," Forest Science 50, 387-397 (2004).

[23] G. Parker (private communication).

[24] R. Makowski (private communication). 